UVX 2008 (2009) 145-149

(C) EDP Sciences, 2009

DOI: $10.1051 / \mathrm{uvx} / 2009023$

\title{
High-resolution scanning coherent X-ray diffraction microscopy
}

\author{
P. Thibault ${ }^{1}$, M. Dierolf ${ }^{1,2}$, A. Menzel ${ }^{1}$, O. Bunk ${ }^{1}$ and F. Pfeiffer ${ }^{1,2}$ \\ ${ }^{1}$ Paul Scherrer Institut, 5232 Villigen PSI, Switzerland \\ ${ }^{2}$ École Polytechnique Fédérale de Lausanne, 1015 Lausanne, Switzerland
}

\begin{abstract}
Scanning transmission x-ray microscopy (STXM) and coherent diffractive imaging (CDI) are imaging techniques that have evolved quite separately. STXM features straightforward data analysis but its resolution is limited by the spot size on the specimen. As for CDI, its promises to reach resolutions below $10 \mathrm{~nm}$ are often hindered by many stringent requirements on the quality of the collected datasets and on the characteristics of the specimen. We describe a ptychographic imaging method that addresses these challenges by bridging the gap between CDI and STXM through the collection of coherent diffraction patterns at each point of a STXM scan. We demonstrate this approach in a hard X-ray experiment, obtaining a five-fold improvement in resolution compared to the focal spot dimensions. The reconstruction algorithm extracts from the data not only the complex-valued transmission function of the specimen but also the complete structure of the wavefield incident on it. The method is expected to allow high-resolution imaging of a wide range of materials and life science specimens.
\end{abstract}

Coherent diffractive imaging (CDI) is the name given to the set of microscopy techniques that rely on coherence to produce high-resolution images. In its simplest form, CDI consists in reconstructing the image of an isolated specimen from the measurement of its far-field diffraction pattern. The method is especially well suited to X-ray microscopy as it effectively removes the need for high-quality highefficiency lenses. With X-rays, the method has been successfully demonstrated on a wide variety of specimens both in 2D and 3D [1-6]. In spite of its successes, CDI is still plagued with important difficulties. Reconstruction of the scattering object involves solving the infamous phase problem which, in many instances, is a computationally difficult inverse problem. In addition, sample preparation is complicated by the requirement that the specimen be completely isolated.

Another powerful microscopy technique, scanning transmission X-ray microscopy (STXM), entails scanning a specimen through a focused X-ray beam and measuring the transmitted intensity at each raster point. Thefingering method is relatively simple and can be conveniently combined with other scanning techniques, such as X-ray fluorescence mapping. However, the resolution of the resulting image is limited by the spot size on the specimen. Increase in resolution thus largely depends on the technological challenge of manufacturing focusing optics.

Here we describe an experimental technique that merges both approaches. The principles of this imaging method were first described a few decades ago by Hegerl and Hoppe [7], who called it "ptychography", and demonstrated since in a few experiments [8-10]. Like STXM, the method involves scanning the specimen with a small beam, and, as CDI, it requires measuring a full diffraction pattern at each raster point. The combination of the two techniques suggests an alternative name for this method: "scanning X-ray diffraction microscopy" (SXDM) [11].

The approach we have adopted to reconstruct a high-resolution image from a ptychography data set is based on the difference map, an iterative algorithm now known to have a wide range of applications going far beyond imaging [12]. Problems solved by the difference map are formulated as a search for the intersection between two constraint sets. A solution attempt is represented by a state vector $\boldsymbol{\Psi}$ which typically satisfies neither constraint until the solution is found. The state vector is updated at each iteration through a combination of projections onto both constraint sets. 
For phase retrieval, $\boldsymbol{\Psi}$ generally is a 2D image or a 3D density. In ptychography, it is the collection of exit waves $\psi_{j}$ corresponding to each position $j$ of the illumination relative to the specimen:

$$
\boldsymbol{\Psi}=\left\{\psi_{1}, \psi_{2}, \ldots, \psi_{N}\right\} .
$$

The two constraints on $\boldsymbol{\Psi}$ are the Fourier and the overlap constraints. The Fourier constraint enforces consistency of each exit wave with its corresponding diffraction pattern. The projection onto this set involves modifying the Fourier magnitudes of each $\psi_{j}$ to be consistent with its corresponding measured data while keeping the phases untouched. The second constraint is given by

$$
\psi_{j}(\boldsymbol{r})=P\left(\boldsymbol{r}-\boldsymbol{r}_{j}\right) O(\boldsymbol{r}),
$$

where $P\left(\boldsymbol{r}-\boldsymbol{r}_{j}\right)$ is the illumination function, called the probe, translated by a known amount $\boldsymbol{r}_{j}$, and $O$ is the specimen's transmission function, called the object. Redundancy in the data, from which ptychographic reconstruction draws its efficiency, is enforced with a scanning step size small enough for adjacent illumination positions to overlap. Each computation of the overlap projection gives a new estimate of the probe and the object as the solution of a coupled system of equations [13]:

$$
\begin{aligned}
& O(\boldsymbol{r})=\frac{\sum_{j} P^{*}\left(\boldsymbol{r}-\boldsymbol{r}_{j}\right) \psi_{j}(\boldsymbol{r})}{\sum_{j}\left|P\left(\boldsymbol{r}-\boldsymbol{r}_{j}\right)\right|^{2}}, \\
& P(\boldsymbol{r})=\frac{\sum_{j} O^{*}\left(\boldsymbol{r}+\boldsymbol{r}_{j}\right) \psi_{j}\left(\boldsymbol{r}+\boldsymbol{r}_{j}\right)}{\sum_{j}\left|O\left(\boldsymbol{r}+\boldsymbol{r}_{j}\right)\right|^{2}} .
\end{aligned}
$$

Unlike previous reconstruction schemes [14,15], prior knowledge of the complex-valued probe profile is not required for the reconstruction to succeed. Instead, the probe is extracted from the dataset along with the image of the specimen.

A schematic of the experimental setup is shown in Figure 1(a). The monochromatic coherent beam is focused with a Fresnel zone plate onto the specimen plane. The exit wave then evolves freely down to a pixel array detector, where its intensity distribution is measured. Examples of such diffraction patterns are given in Figure $1(b)$. The high-resolution image of the specimen is then reconstructed with the help of a phase retrieval algorithm. We have tested the method on a test specimen consisting of a zone plate buried under a $\sim 1 \mu$ m layer of gold. The experiment was conducted at the cSAXS beamline of the Swiss Light Source, Paul Scherrer Institut, Switzerland. A spatially coherent portion

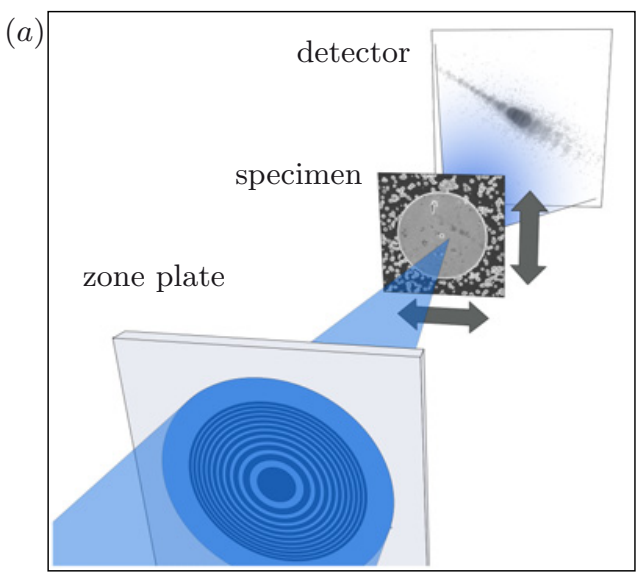

$(b)$
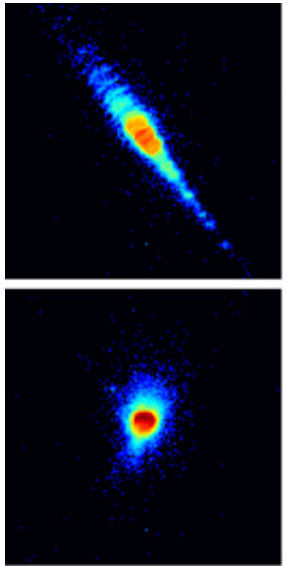
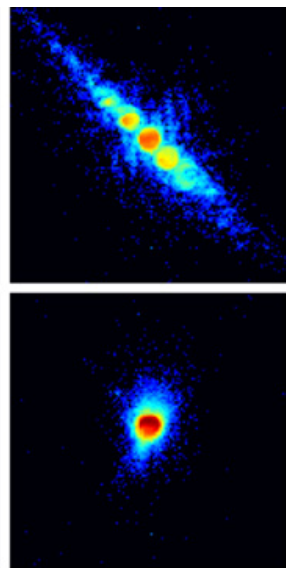

Figure 1. (a) Schematic of the experimental setup. The beam is focused by a Fresnel zone plate. Past the specimen the wave propagates freely to the detector, where its far-field intensity pattern is measured. $(b)$ Examples of diffraction patterns obtained in this type of experiment. 
of the hard X-ray beam $(6.8 \mathrm{keV})$ was selected by a $20 \mu$ m diameter aperture placed before the zone plate. The specimen was mounted on a piezo-electric translation stage. The flux on the specimen was approximately $1.2 \times 10^{7}$ photons/s. Diffraction patterns were measured with the Pilatus $2 \mathrm{M}$, a singlephoton counting detector having no readout noise and no dark-current. Each diffraction pattern covers a solid angle of about $10 \times 10 \mathrm{mrad}^{2}$ and is the result of a $50 \mathrm{~ms}$ exposure.

A preliminary evaluation of the data can be obtained immediately by a STXM-like analysis of the dataset. At each point in the raster scan, the total intensity provides a low-resolution estimate of the absorption of the specimen, giving the map shown in Figure 2(b). The first moments in $x$ and $y$ of the measured intensity distributions, shown in Figures $2(c-d)$, give additional information in the form of differential phase contrast images.
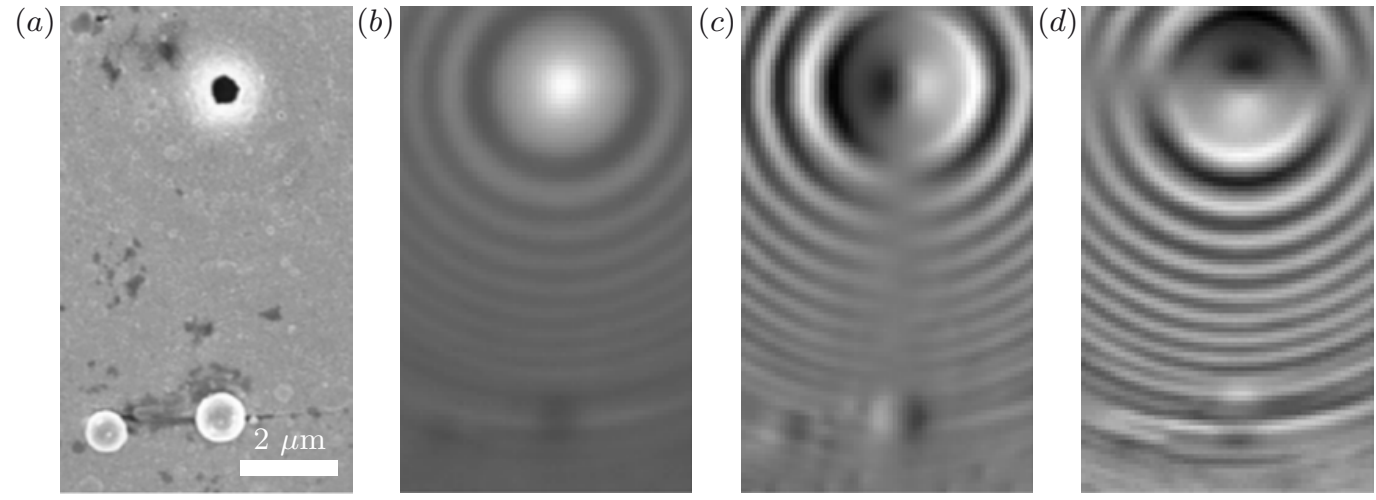

Figure 2. (a) Scanning electron micrograph (SEM) of the scanned region. $(b-d)$ Preliminary analysis of the data. (b) Transmission map obtained by integrating the total transmitted intensity in each diffraction pattern. $(c, d)$ First moment of the measured intensity distribution in $x$ and in $y$, respectively, giving the gradient of the phase part of the transmission function. Images $(b-d)$ require little computation and can be used to provide immediate feedback to the microscopist.
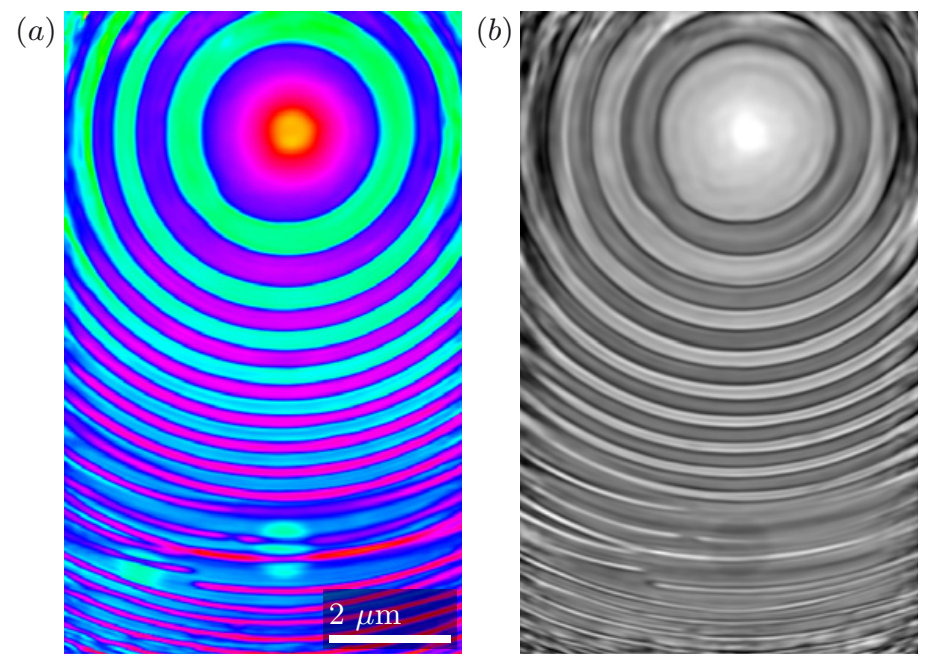

Figure 3. SXDM reconstruction of a portion of the zone plate specimen, using $50 \times 90$ diffraction patterns. $(a)$ Phase and (b) Absorption. Comparison with Figure 2 demonstrates the clear enhancement in the resolution. The reconstruction is quantitative: both the phase and the amplitude are in excellent agreement with the expected phase shift and absorption of the wave as it traverses the specimen. 
The high-resolution image of the specimen, shown in Figure 3, is reconstructed using the iterative algorithm described above. The image is complex-valued since it represents the complex index of refraction of the specimen integrated along the direction of propagation. In total, $50 \times 90$ diffraction patterns are used for this reconstruction, each sampled on a $128 \times 128$ array, giving a real-space pixel size of $18 \mathrm{~nm}$. Convergence of the iterative algorithm was observed after only a few tens of iterations. To ensure reproducibility, the image shown in Figure 3 is the average of 30 reconstruction estimates taken every 5 iterations along the same run once the algorithm had converged.

The incident wavefield is recovered in addition to the specimen's image. Compared to methods relying on a model, retrieval of the probe can improve substantially the quality of the reconstructed image. In the experiment discussed here, the reconstructed probe, Figure 4(a), featured a strong phase curvature, indicating that the specimen was placed downstream from the focal plane. Propagating the probe numerically back to the focal plane, Figure $4(b)$, the defocus distance was found to be about $1.2 \mathrm{~mm}$. The possibility of reconstructing the incoming wavefield suggests that SXDM can act as an efficient wavefront sensing method to characterize accurately the performance of X-ray optics.

$(a)$

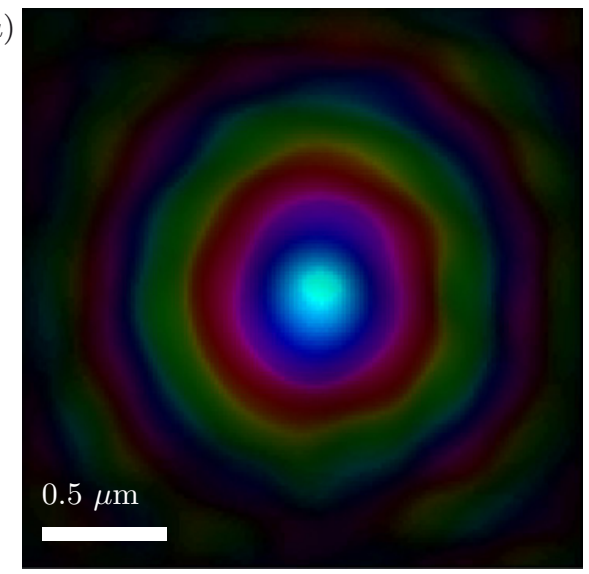

(b)

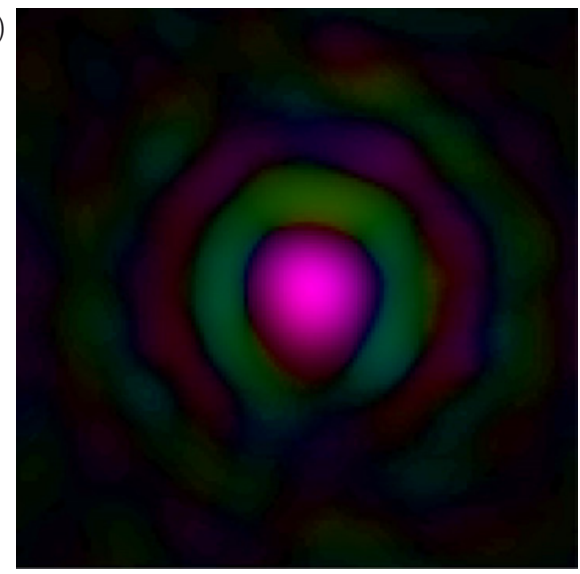

Figure 4. (a) Reconstructed probe in the specimen plane. (b) The same probe propagated by $1.2 \mathrm{~mm}$ back to the focal plane. In these complex-valued images, the phase part is mapped to hue, and the amplitude part is mapped to brightness.

Our current research program aims at obtaining higher resolutions by increasing the highest significant scattering angle through longer exposures. We also expect SXDM to work successfully with other focusing devices, such as reflective and compound refractive optics. Extension of the method to three-dimensional imaging, through tomographic reconstructions or more integrated reconstruction approaches, is also envisaged.

\section{References}

[1] J. Miao, P. Charalambous, J. Kirz, D. Sayre, Nature 400, 342 (1999)

[2] G.J. Williams, M.A. Pfeifer, I.A. Vartanyants, I.K. Robinson, Phys. Rev. Lett. 90(175501), 1 (2003)

[3] S. Eisebitt, J. Luning, W.F. Schlotter, M. Lorgen, O. Hellwig, W. Eberhardt, J. Stohr, Nature 432(7019), 885 (2004)

[4] D. Shapiro, P. Thibault, T. Beetz, V. Elser, M.R. Howells, C. Jacobsen, J. Kirz, E. Lima, H. Miao, A.M. Nieman et al., Proc. Natl. Acad. Sci.USA 102(43), 15343 (2005) 
[5] H.N. Chapman, A. Barty, S. Marchesini, A. Noy, C. Cui, M.R. Howells, R. Rosen, H. He, J.C.H. Spence, U. Weierstall et al., J. Opt. Soc. Am. A 23(5), 1179 (2006)

[6] H.N. Chapman, A. Barty, M.J. Bogan, S. Boutet, M. Frank, S.P. Hau-Riege, S. Marchesini, B.W. Woods, S. Bajt, W.H. Benner et al., Nature Physics 2(12), 839 (2006)

[7] R. Hegerl, W. Hoppe, Ber. Bunsen-Ges. Phys. Chem. 74, 1148 (1970)

[8] H.N. Chapman, Ultramicroscopy 66, 153 (1996)

[9] J.M. Rodenburg, A.C. Hurst, A.G. Cullis, Ultramicroscopy 107, 227 (2007)

[10] J.M. Rodenburg, A.C. Hurst, B.R. Dobson, F. Pfeiffer, O. Bunk, C. David, K. Jefimovs, I. Johnson, Phys. Rev. Lett. 98(034801), 1 (2007)

[11] P. Thibault, M. Dierolf, A. Menzel, O. Bunk, C. David, F. Pfeiffer, Science 321, 379 (2008)

[12] V. Elser, I. Rankenburg, P. Thibault, Proc. Natl. Acad. Sci.USA 104(2), 418 (2007)

[13] P. Thibault, M. Dierolf, A. Menzel, O. Bunk, F. Pfeiffer (2008), in review

[14] J.M. Rodenburg, R.H.T. Bates, Phil. Trans. R. Soc. Lond. A 339(1655), 521 (1992)

[15] J.M. Rodenburg, H.M.L. Faulkner, Applied Physics Letters 85, 4795 (2004) 